\title{
Characteristics Research on Self-Amplified Distributed Feedback Fiber Laser
}

\author{
Zhiqiang $\mathrm{SONG}^{1 *}$, Haifeng $\mathrm{QI}^{1}$, Jian $\mathrm{GUO}^{1}$, Chang $\mathrm{WANG}^{1}$, and Gangding $\mathrm{PENG}^{2}$ \\ ${ }^{1}$ Shandong Key Laboratory of Optical Fiber Sensing Technologies, Laser Institute of Shandong Academy of Sciences, \\ Jinan, 250014, China \\ ${ }^{2}$ School of Electrical Engineering and Telecommunications, University of New South Wales, Sydney, Australia 2052 \\ *Corresponding author: Zhiqiang SONGＩE-mail: zhiqiangs@gmail.com
}

\begin{abstract}
A distributed feedback (DFB) fiber laser with a ratio of the backward to forward output power of 1:100 was composed by a 45-mm-length asymmetrical phase-shifted fiber grating fabricated on the 50-mm erbium-doped photosensitive fiber. Forward output laser was amplified using a certain length of Nufern EDFL-980-Hp erbium-doped fiber to absorb the surplus pump power after the active phase-shifted fiber grating and get population inversion. By using OptiSystem software, the best fiber length of the EDFL to get the highest gain was simulated. In order to keep the amplified laser with the narrow line-width and low noise, a narrow-band light filter consisting of a fiber Bragg grating (FBG) with the same Bragg wavelength as the laser and an optical circulator was used to filter the amplified spontaneous emission (ASE) noise of the out-cavity erbium-doped fiber. The designed laser structure sufficiently utilized the pump power, and a DFB fiber laser with the $32.5-\mathrm{mW}$ output power, $11.5-\mathrm{kHz}$ line width, and $-87-\mathrm{dB} / \mathrm{Hz}$ relative intensity noise (RIN) at $300 \mathrm{~mW}$ of $980 \mathrm{~nm}$ pump power was brought out.
\end{abstract}

Keywords: Distributed feedback fiber laser, self-amplification, narrow-linewidth, low-noise

Citation: Zhiqiang SONG, Haifeng QI, Jian GUO, Chang WANG, and Gangding PENG, "Characteristics Research on Self-Amplified Distributed Feedback Fiber Laser," Photonic Sensors, 2014, 4(3): 265-268.

\section{Introduction}

Distributed feedback fiber laser (DFB-FL) is a kind of laser consisting of a single phase shifted fiber grating directly writing in the rare earth doped fiber, depending on the narrow bandwidth, low noise, and stable single-mode operation, and it has the important application value as high sensitivity sensors in the acoustic, vibration signal detection technologies [1, 2]. Nevertheless, due to the very short cavity length and not enough high rare earth ions concentration, the output power of the DFB-FL is small, which makes it obvious that it cannot fully meet the requirements of applications such as coherent detection, laser radar, despite with the coherence length of a few hundred kilometers. The use of the $\mathrm{Er} / \mathrm{Yb}$ co-doped fiber [3] and master oscillator power-amplifier (MOPA) structure [4] is an effective method to improve the fiber laser conversion efficiency. Considering the gain fiber of the DFB-FL is very short and the vast majority of the pump's power will not be absorbed, a self-amplification structure DFB-FL was designed. Without the additional pump source, only a suitable length erbium-doped fiber connected on the fore-end of the DFB-FL was used to absorb the residual pump power and achieve population inversion, so

Received: 13 December 2013 / Revised version: 5 January 2014

C The Author(s) 2014. This article is published with open access at Springerlink.com DOI: $10.1007 / \mathrm{s} 13320-014-0170-7$

Article type: Regular 
the forward output laser would be amplified. In order to keep the amplified laser with the narrow line-width and low noise, a narrow-band light filter consisting of a fiber Bragg grating (FBG) with the same Bragg wavelength of the laser and an optical circulator was used to filter the amplified spontaneous emission (ASE) noise of the out-cavity erbium-doped fiber. Finally, a 30-mW DFB-FL with the narrow linewidth and low noise was employed.

\section{Experiment and discussion}

\subsection{Fabrication of asymmetric structure DFB-FL}

The phase-shifted fiber Bragg grating (PS-FBG) is the core device of the DFB-FL. There are a variety of its fabrication methods, such as the shielding method and double exposure method, and we adopted the phase mask moving method [5] to make a more accurate PS-FBG in an asymmetric structure. It had the structure with a $45-\mathrm{mm}$ grating length on a 50-mm Nufern PS-ESF-3/125 photosensitive erbium-doped fiber as shown in Fig. 1. In order to improve the one-way output laser, the phase shift was introduced in the $0.4 L_{g}$ position, the transmission window wavelength of the phase-shifted grating was $1536.1 \mathrm{~nm}$, and the grating coupling coefficient was about $150 \mathrm{~m}^{-1}$.

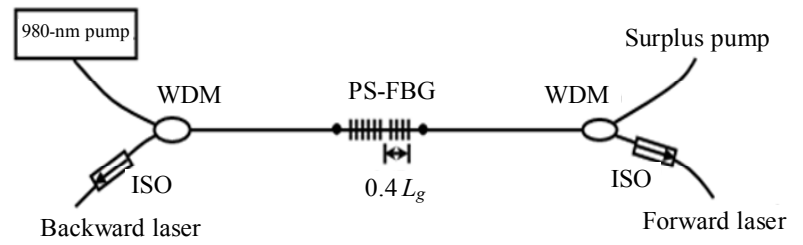

Fig. 1 Structure of the asymmetric DFB-FL.

Using the 980-nm laser diode (LD) as the pump source, the pump input from the far-end from phase shift point, the output power, and the residual pump power are shown in Fig. 2. It can be seen that the ratio of the backward to forward output power was over 1:100, and the laser had the good one-way output. The forward laser power was about $320 \mu \mathrm{W}$ at the $300-\mathrm{mW}$ pump, the slope efficiency was above $0.1 \%$, and the surplus pump power through the PS-FBG still reached $200 \mathrm{~mW}$. Using the null method of measurement as shown in Fig. 3, the DFB-FL's linewidth and relative intensity noise (RIN) delay were $6.7 \mathrm{kHz}$ and $-102 \mathrm{~dB} / \mathrm{Hz}$, the laser signal-to-noise ratio was $65 \mathrm{~dB}$, and the data are collected in Table 1 for the further discussion.

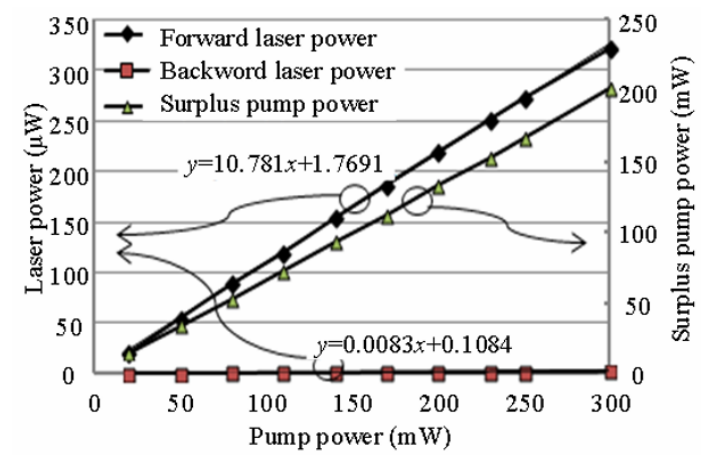

Fig. 2 Output power of the asymmetric DFB-FL.

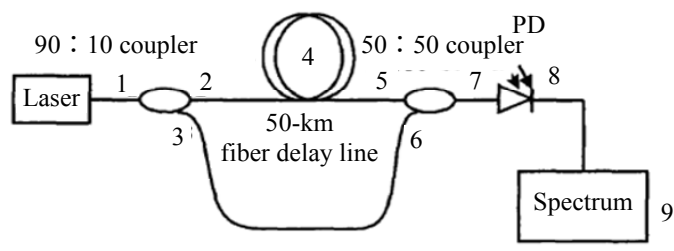

Fig. 3 Setup of linewidth measurement with null method.

Table 1 Comparison of performance before and after DFB-FL amplification.

\begin{tabular}{ccccccc}
\hline & $\begin{array}{c}\text { Power } \\
(\mathrm{mW})\end{array}$ & $\begin{array}{c}\text { Line-width } \\
(\mathrm{kHz})\end{array}$ & $\begin{array}{c}\text { RIN } \\
(\mathrm{dB} / \mathrm{Hz})\end{array}$ & $\begin{array}{c}\text { Efficiency } \\
(\%)\end{array}$ & $\begin{array}{c}\text { SNR } \\
(\mathrm{dB})\end{array}$ & $\begin{array}{c}\text { Surplus } \\
\text { pump } \\
\text { power } \\
(\mathrm{mW})\end{array}$ \\
\hline $\begin{array}{c}\text { Original } \\
\text { DFB-FL }\end{array}$ & 0.32 & 6.7 & -102 & 0.1 & 65 & 201 \\
$\begin{array}{c}\text { Self-amplified } \\
\text { DFB-FL }\end{array}$ & 32.5 & 11.5 & -87 & 11.9 & 75 & 30 \\
\hline
\end{tabular}

\subsection{Amplification of erbium-doped fiber length optimization}

The DFB-FL cannot make full use of the pump, and if adding a certain length erbium-doped fiber out-cavity, an Erbium doped fiber amplifier (EDFA) can be formed to amplify the laser signal, as shown in Fig. 4. Because the signal light and pump light were all produced from the DFB-FL, the value of the power corresponded to the forward laser power and the residual pump power at the certain pump power, as shown in Fig. 2, so the signal power and pump power of the EDFA could not change alone, 
but in accordance with the DFB-FL power curve.

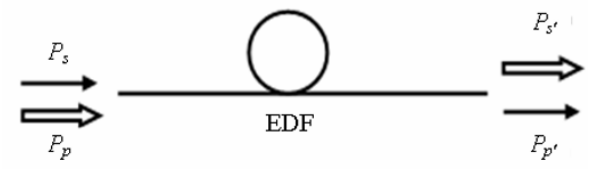

Fig. 4 Model of DFB-FL amplification.

The simulation results of the gain of laser with the length of the Er-doped fiber at $100 \mathrm{~mW}, 200 \mathrm{~mW}$, and $300 \mathrm{~mW}$ are shown in Fig. 5. The Er-doped fiber parameters accorded with the performances of Nufern EDFL980-Hp whose concentration of $\mathrm{Er}^{3+}$ was $4000 \mathrm{ppm}$. As it can be seen, the signal gain did not continue to grow with an increase in the Er-doped fiber length, but there was an optimum fiber length to maximize the gain. According to the simulation results, the optimum length of EDFL980-Hp to produce the maximum gain of the above-mentioned DFB-FL was $1.5 \mathrm{~m}$, and the maximum gain researched $25 \mathrm{~dB}$ based on the $320-\mu \mathrm{W}$ laser single power at a pump power of $300 \mathrm{~mW}$.

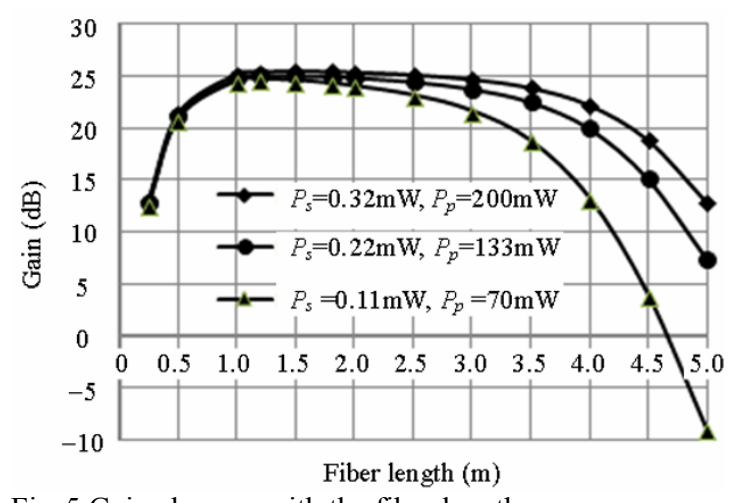

Fig. 5 Gain changes with the fiber length.

\subsection{Amplification and filter structure of DFB-FL}

According to the simulation results, using the 1.5-m Nufern EDFL980-Hp fiber as the out-cavity gain fiber of the DFB-FL, in order to eliminate the influence of ASE of the erbium doped fiber on the laser performance, a filter consisting of an FBG with the same Bragg wavelength of the laser and an optical circulator was used. The diagram of the DFB-FL which had the structure of selfamplification and the filter is shown in Fig. 6, in which the FBG and PS-FBG were placed in the same block heat sink, the FBG had the reflection bandwidth of $0.2 \mathrm{~nm}$, side mode suppression of $15 \mathrm{~dB}$, and reflectance ratio of greater than $99 \%$.

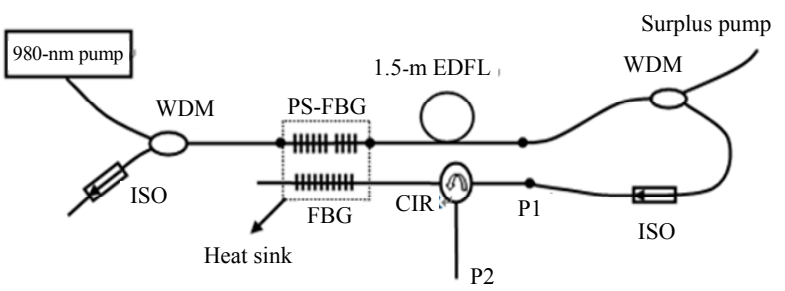

Fig. 6 Structure of the self-amplification DFB-FL.

The power curve and spectrum before and after filtering measured at P1 and P2 are shown in Figs. 7 and 8 , respectively. Compared to the original DFB-FL, the laser slope efficiency increased significantly after being amplified by the out-cavity Er-doped fiber. The output laser power reached $45.5 \mathrm{~mW}$ without filtering, more than $32.5 \mathrm{~mW}$ with filtering the ASE noise under the $300-\mathrm{mW}$ pump power, and the slope efficiency did not have a trend of diminution. If continuing to improve the pump power, the laser output power could increase. Compared to the $0.32-\mathrm{mW}$ original laser signal, the self-amplification laser achieved another gain of $20 \mathrm{~dB}$, but the value was much less than the value of the simulation results, and this is due to the effect of losses and ASE noise which were not considered in the model. As it can be seen in Fig. 7, the signalto-noise ratio was only $50 \mathrm{~dB}$ without filtering, slightly higher than the original DFB-FL noise suppressed by the FBG filter.

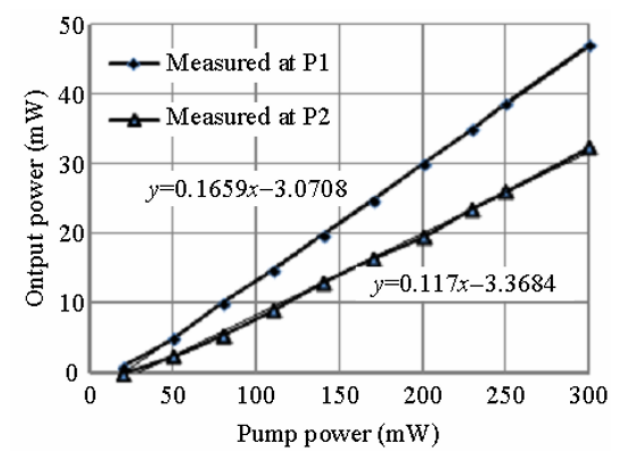

Fig. 7 Output power of the self-amplification DFB-FL.

The linewidth and RIN of the self-amplification laser were $11.5 \mathrm{kHz}$ and $-87 \mathrm{~dB} / \mathrm{Hz}$, the linewidth was broadened with a 2-fold increase, and the RIN 
decreased by $15 \mathrm{~dB}$ compared to the original DFB-FL signal, but the laser slope efficiency increased to $11.9 \%$ from $0.1 \%$. Being benefited from an increase in the output power and the suppression of the background noise, the signal-tonoise ratio increased by nearly $10 \mathrm{~dB}$.

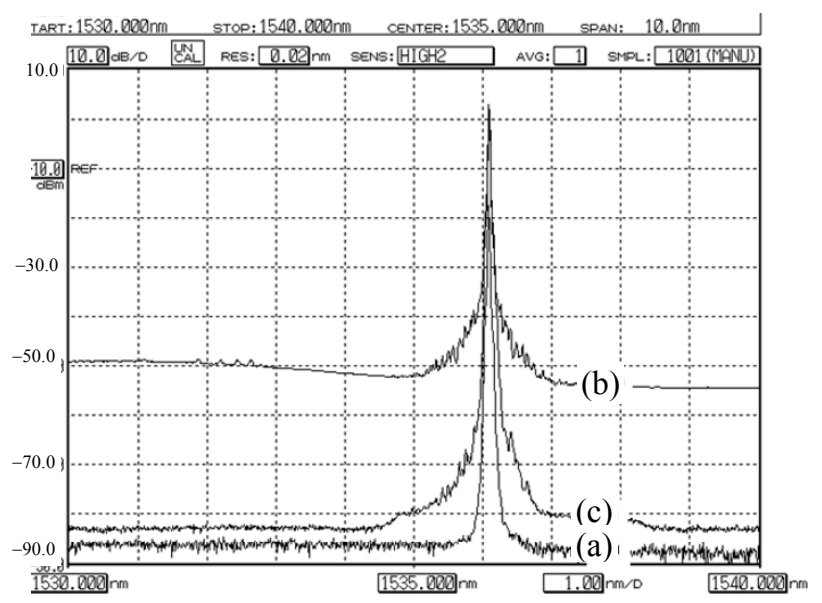
(c) P2.

Fig. 8 Laser spectra of (a) original DFB-FL, (b)P1, and

\section{Conclusions}

The narrow linewidth fiber laser is one of the key development directions of the fiber laser technology, and it will further widen the application field by obtaining the higher output power at the same time, ensuring the narrow linewidth and low noise characteristics. Combining the DFB-FL with the optical fiber amplifier, the self-amplification DFB-FL was designed. The one-way output structure of the DFB-FL provided a low noise and narrow linewidth laser signal, and the reasonable out-cavity Er-doped fiber effectively utilized the residual pump power and provided more gain of the laser signal. And the introduction of the fiber Bragg grating filter ensured the characteristics of the amplification low noise and narrow linewidth laser output. This provides a simple and feasible structure scheme for the realization of the narrow linewidth fiber laser with the larger power.

Open Access This article is distributed under the terms of the Creative Commons Attribution License which permits any use, distribution, and reproduction in any medium, provided the original author(s) and source are credited.

\section{References}

[1] A. Tikhomirov and S. Foster, "DFB FL sensor crosscoupling reduction," Journal of Lightwave Technology, 2007, 25(2): 533-538.

[2] S. Xiong, W. Liu, and H. Yang. "Investigation on the coherence collapse characteristics of single-longitudinal-mode DFB fiber laser," Laser \& Optoelectronics Progress, 2013, 50(2): 161-166.

[3] K. Yelen, L. Hickey, and M. Zervas. "Experimentally verified modeling of erbium-ytterbium co-doped DFB fiber lasers," Journal of Lightwave Technology, 2005, 23(3): 1380-1392.

[4] N. Y. Voo, J. K. Sahu, and M. Ibsen, "345-mW 1836-nm single-frequency DFB fiber laser MOPA," IEEE Photonics Technology Letters, 2005, 17(12): 2550-2552.

[5] H. Qi, Z. Song, and S. Li, "Apodized distributed feedback fiber laser with asymmetrical outputs for multiplexed sensing applications," Optics Express, 2013, 21(9): 11309-11314. 